\title{
Cosmic-ray and X-ray Heating of Interstellar Clouds and Protoplanetary Disks
}

\author{
Alfred E. Glassgold \\ Astronomy Department, University of California, Berkeley, CA 94720-3411, USA \\ aglassgold@berkeley .edu \\ Daniele Galli \\ INAF-Osservatorio Astrofisico di Arcetri, Largo E. Fermi 5, 50125, Italy \\ galli@arcetri.astro.it \\ and \\ Marco Padovani \\ Laboratoire de Radioastronomie Millimétrique, UMR 8112 du CNRS, École Normale \\ Supérieure et Observatoire de Paris, 24 rue Lhomond, 75231 Paris cedex 05 \\ marco.padovani@lra.ens.fr
}

Received 2012 May 14; accepted 2012 July 20 


\begin{abstract}
Cosmic-ray and X-ray heating are derived from the electron energy loss calculations of Dalgarno, Yan and Liu for hydrogen-helium gas mixtures. These authors treated the heating from elastic scattering and collisional de-excitation of rotationally excited hydrogen molecules. Here we consider the heating that can arise from all ionization and excitation processes, with particular emphasis on the reactions of cosmic-ray and X-ray generated ions with the heavy neutral species, which we refer to as chemical heating. In molecular regions, chemical heating dominates and can account for $50 \%$ of the energy expended in the creation of an ion pair. The heating per ion pair ranges in the limit of negligible electron fraction from $\sim 4.3 \mathrm{eV}$ for diffuse atomic gas, to $\sim 13 \mathrm{eV}$ for the moderately dense regions of molecular clouds and to $\sim 18 \mathrm{eV}$ for the very dense regions of protoplanetary disks. An important general conclusion of this study is that cosmic-ray and X-ray heating depends on the physical properties of the medium, i.e., on the molecular and electron fractions, the total density of hydrogen nuclei, and to a lesser extent on the temperature. It is also noted that chemical heating, the dominant process for cosmic-ray and X-ray heating, plays a role in UV irradiated molecular gas.
\end{abstract}

\title{
1. Introduction
}

There has been a significant increase of interest in the cosmic-ray ionization rate in both

local and distant neighborhoods. Much of it has been spurred by infrared absorption-line measurements of the $\mathrm{H}_{3}^{+}$ion in diffuse and translucent interstellar clouds (e.g., Indriolo et al. 2007 and references therein). Indriolo \& McCall (2012) have extended these measurements 
to fifty lines of sight dominated by translucent clouds with more than one magnitude of visual extinction, obtaining 21 detections. Their straightforward analysis of the observations yields values of the ionization rate for $\mathrm{H}_{2}$ in the range $\sim(2-11) \times 10^{-16} \mathrm{~s}^{-1}$, significantly larger than the range of values that had been in use for several decadest 1 . This result has led to the reconsideration of how both electron and proton cosmic-rays propagate in the interstellar medium (e.g., Indriolo et al. 2009, Padovani et al. 2009, Padovani \& Galli 2011, Rimmer et al. 2012, Everett \& Zweibel 2011). In the context of the new survey, Indriolo \& McCall al. (2012) reviewed the explanation advanced by Indriolo et al. (2009) and Padovani et al. (2009) that the intensity of low-energy protons is reduced inside dense molecular clouds. The large difference between diffuse/translucent and dense clouds is maintained in Indriolo \& McCall (2012), but their sample shows little evidence for a dependence of ionization rate on column density. The value of the cosmic ray ionization rate for the local interstellar medium remains an open question.

Another topic of interest relating to the interaction of cosmic-rays with the interstellar medium is their role in heating. Cosmic-rays are an efficient (often dominant) source of heating in various environments, from the dense gas in molecular clouds (Goldsmith \& Langer 1978), both in normal and starbust galaxies (e.g., Suchkov et al. 1993), to photodissociation regions (Shaw et al. 2009), and possibly even in the primordial gas (Jasche et al. 2007). In dense, shielded regions like molecular cloud cores, the balance of cosmicray heating and gas cooling determines a temperature gradient decreasing inwards (Galli et al. 2002) that has been accurately traced by interferometric observations of molecular emission in prestellar cores (Crapsi et al. 2007, Pagani et al. 2007) and dark globules (Pineda $\&$ Bensch 2007). The values for the cosmic-ray heating energy per ion pair available in the

\footnotetext{
${ }^{1}$ In this and other papers, the cosmic-ray ionization rate $\zeta_{\mathrm{H}}$ per $\mathrm{H}$ nucleus is used, which is smaller by a factor of two than the rate $\zeta_{\mathrm{H}_{2}}$ per $\mathrm{H}_{2}$ molecule of Indriolo et al. (2007).
} 
literature range over a factor of three. In this paper we will attempt to clarify this situation for both cosmic-ray and X-ray heating.

The interaction of fast electrons slowing down in molecular gas was first analyzed more than three decades ago by Glassgold \& Langer (1973, hereafter GL73), Cravens et al. (1975) and Cravens \& Dalgarno (1978). Although GL73 was the first paper to consider cosmic ray heating in interstellar molecular regions, it suffered from the incompletely known electron cross sections of the early 70s, a crude energy-loss calculation, and cumbersome notation. Cravens et al. (1975) did a better job on cross sections and energy loss, but restricted themselves to low-energy electrons in order to evaluate the validity of the commonly used continuous slowing down approximation. This limitation was removed by Cravens \& Dalgarno (1978). There are no glaring discrepancies between Cravens \& Dalgarno (1978) and GL73. All of these papers considered pure $\mathrm{H}_{2}$ regions, i.e., the roles of $\mathrm{H}^{+}$and $\mathrm{He}^{+}$ions in molecular gas were ignored. A more up to date and complete analysis was carried out by Dalgarno et al. (1999, hereafter DYL). DYL considered carefully all of the energy loss channels for electron energies from $30 \mathrm{eV}$ to $1 \mathrm{keV}$ in various mixtures of $\mathrm{H}, \mathrm{H}_{2}$ and $\mathrm{He}$. They showed how the energy expended to make an ion pair $W$ is partitioned between elastic and non-elastic processes, but they did not fully treat the heating.

Cosmic-rays and X-rays (or the equivalent, fast electrons) produce ions and excited molecules that can interact with the dominant neutral atomic or molecular gas. The products of these reactions carry away a significant amount of the available energy and heat the gas. We refer to this as chemical heating, and quantify it by a quantity $Q_{\text {chem }}$ defined per ion pair. Chemical heating by cosmic-rays and X-rays occurs in a wide range of applications from diffuse and dense interstellar clouds to pre-stellar cores, protoplanetary disks, and planetary atmospheres. In this paper we use the results of DYL to inquire how the excited species and ions heat a gas mixture of $\mathrm{H}, \mathrm{H}_{2}$, and $\mathrm{He}$. We will show that $\sim 1 / 2$ of the energy of X-rays 
and cosmic rays can go into heating in dense molecular regions and that chemical heating can be the most important part of the heating. We also note that chemical heating occurs when molecular regions are irradiated by far ultra-violet (FUV) photons.

This paper is organized as follows. In Section 2 we outline the physical basis of our analysis and make quantitative estimates in Sections 3 and 4 . We discuss the most important results in Section 5. The paper ends with a short summary in Section 6.

\section{The Physical Basis for Cosmic-ray and X-ray Heating}

A basic tenet of earlier work on cosmic-ray heating is that proton cross sections can be represented accurately by the corresponding ones for electrons (which are generally more available), at least at sufficiently large energy. Cravens \& Dalgarno (1978) discussed this explicitly on the basis of the good agreement between experiments on proton collisional ionization of $\mathrm{H}_{2}$ (Hooper et al. 1961) and the Born approximation, as developed by Bates \& Griffing (1953), all the way down to a proton energy of $0.06 \mathrm{MeV}$. This conclusion stems from the fact that high-energy ionization cross sections are a function of the incident particle's speed, so proton cross sections are the same as those for electrons with the same speed, i.e., when the electron energy $E$ satisfies the relation $E=\left(m_{\mathrm{e}} / m_{\mathrm{p}}\right) E_{\mathrm{p}}$.

An energetic cosmic-ray proton loses energy in cosmic gases in small steps that are characterized by the energy $W$ to make an ion pair, known to be close to $37 \mathrm{eV}$ from extensive experimental and theoretical studies. In their interactions with the hydrogen and helium of the gas, each cosmic-ray (proton) ionization generates a distribution of relatively slow electrons with average energy of $19 \mathrm{eV}$ (Opal et al. 1971; GL73) and that leads to another "secondary" ionization two-thirds of the time. X-rays with keV energies develop a similar sequence once the initial photon is absorbed by a heavy atom by L- or K-shell electron 
ejection. The energy of this primary electron is $E=h \nu_{X}-B-\mathcal{E}$, where $B$ is the binding energy of the $\mathrm{K}$ or $\mathrm{L}$ shell of the heavy atom and $\mathcal{E}$ is the mean energy of the resulting ion. It is significantly less than the initial X-ray energy, but much of the difference is restored in the form of several prompt supra-thermal Auger electrons with keV energies. There is also some X-ray fluorescence that, by the processes just described, generates more fast electrons. The net effect of X-ray ionization of heavy atoms, accompanied by Auger electrons and fluorescence, is that most of the initial X-ray photon energy is recovered in the form of the primary electron plus several other fast electrons. Thus, once the the processes attending the absorption of a keV X-ray have occurred, the main ionization effects of the X-ray are accomplished by the fast electrons that it produces. This is almost exactly the situation with cosmic-ray ionization, except that these electrons are replaced by the cosmic-ray proton which, as noted above, behaves like an electron of the same speed. This is the basis for our using the extensive results of DYL on the interaction of electrons with atomic and molecular gases to calculate the heating by cosmic-rays and X-rays.

The spectra of cosmic-rays and X-rays can change from region to region due to proximity to a source or absorption within the cloud of interest. One example is the absorption of low-energy protons in thick clouds, proposed by Indriolo et al. (2009) and Padovani et al. (2009) to explain the reduction of the ionization rate inside dense molecular clouds. Another is the observed variation in the observed spectra of the X-rays emitted by young stellar objects. DYL give results for mono-energetic electrons with energies from 30 to $1000 \mathrm{eV}$. Their results do not vary by more than $10 \%$ for $E>200 \mathrm{eV}$, and if the underlying spectrum does not extend below this energy for electrons or the equivalent energy of $0.4 \mathrm{Mev}$ for protons, the variation of the DYL results at low electron energy can be ignored. Otherwise the results presented in this paper have to be integrated over the appropriate spectrum. Below $E=30 \mathrm{eV}$ (or $\sim 60 \mathrm{keV}$ protons), the lowest energy considered by DYL, one can use GL73, but the equivalence between electrons and protons has very likely broken down at this 
point. In this paper, we focus on electrons with energies greater than $500 \mathrm{eV}$, corresponding to protons with energies greater than $1 \mathrm{MeV}$.

We use DYL to analyze all of the outcomes of a fast electron as it slows down by interacting with a cosmic gas-mixture dominated by $\mathrm{H}, \mathrm{H}_{2}$, and $\mathrm{He}$, ignoring the much less abundant heavy elements. Unlike DYL, we make a comprehensive effort to evaluate how much of the energy expended in each process goes into heating - the primary goal of this paper. For this purpose, the most useful results in DYL are Table 4 (ion production), Table 5 (excitation), and Table 7 (heating efficiency), all for the case of $\mathrm{H}, \mathrm{He}$ and $\mathrm{H}_{2}, \mathrm{He}$ gas mixtures.

The energy partitions for a $1-\mathrm{keV}$ electron in a $\mathrm{He}, \mathrm{H}_{2}$ mixture in the limit of zero electron fraction are listed in Table 1. The entries are independent of energy above 100-200 $\mathrm{eV}$. If the X-ray spectrum extends below $200 \mathrm{eV}$ or the cosmic-ray spectrum below $400 \mathrm{keV}$, the full tables in DYL contain the information needed for lower energies. Table 1 has been organized into four parts: ion production; excitation of $\mathrm{H}_{2}$ levels; excitation of He levels; and the heating from elastic scattering combined with the heating from rotational excitation. All energies are expressed in $\mathrm{eV}$. The first column names the process; the second column the energy expended for that process $W$; the third column the number of such processes for an electron energy $E, N=E / W$, whe the electron energy is $E=1 \mathrm{keV}$; the fourth column the threshold energy $E_{\text {th }}$ for the process; and lastly the energy associated with the process, i.e., the product $N E_{\text {th }}$ of the two previous columns (for $E=1 \mathrm{keV}$ ).

For example the first four lines of Table 1 show that a keV electron generates $23.9 \mathrm{H}_{2}^{+}$ ions, $1.1 \mathrm{H}^{+}$ions, $2.2 \mathrm{He}^{+}$ions, and $<0.1 \mathrm{He}^{2+}$ ions, for a total of 27.2 ions. This follows from the fact that an incident electron expends on average an energy $W_{i}$ to make an ion pair of type $i\left(i=1\right.$ for $\mathrm{H}_{2}^{+}, i=2$ for $\mathrm{H}^{+}, i=3$ for $\mathrm{He}^{+}$, and $i=4$ for $\left.\mathrm{He}^{2+}\right)$. More generally, if $N_{i}=E / W_{i}$ is the number of ions generated of type $i$, the total number of ions 
Table 1. DYL Energy Partitions for a $\mathrm{He}, \mathrm{H}_{2}$ mixture ${ }^{\mathrm{a}}$

\begin{tabular}{|c|c|c|c|c|}
\hline Process & $\begin{array}{c}W \\
(\mathrm{eV})\end{array}$ & $N$ & $\begin{array}{l}E_{\mathrm{th}} \\
(\mathrm{eV})\end{array}$ & $\begin{array}{c}N E_{\mathrm{th}} \\
(\mathrm{eV})\end{array}$ \\
\hline $\mathrm{H}_{2}^{+}$ & 41.9 & 23.87 & 15.44 & 369 \\
\hline $\mathrm{H}^{+}$ & 921 & 1.09 & 18.08 & 20 \\
\hline $\mathrm{He}^{+}$ & 459 & 2.18 & 24.59 & 54 \\
\hline $\mathrm{He}^{2+}$ & 16000 & 0.0625 & 54.42 & 3 \\
\hline Total Ion production & & & & 446 \\
\hline B & 117 & 8.55 & 11.37 & 97 \\
\hline $\mathrm{C}$ & 132 & 7.58 & 12.42 & 94 \\
\hline Dissociation $^{\mathrm{b}}$ & 92.6 & 10.8 & $\sim 13$ & 140 \\
\hline $\mathrm{H}+\mathrm{H}(2 p)$ & 534 & 1.87 & 14.7 & 28 \\
\hline$v=1$ & 7.81 & 128 & 0.516 & 66 \\
\hline$v=2$ & 109 & 9.17 & 1.032 & 10 \\
\hline Total $\mathrm{H}_{2}$ excitation & & & & 435 \\
\hline He $2^{1} \mathrm{~S}^{\mathrm{c}}$ & 15000 & 0.0667 & 20.62 & 1 \\
\hline $2^{1} \mathrm{P}$ & 1940 & 0.515 & 21.22 & 11 \\
\hline $2^{3} \mathrm{~S}$ & 34900 & 0.0287 & 19.82 & 1 \\
\hline $2^{3} \mathrm{P}+n>2^{3}$ & 22500 & 0.0444 & 21.0 & 1 \\
\hline$n>2^{1}$ & 3520 & 0.284 & 23 & 7 \\
\hline Total He excitation & & & & 21 \\
\hline Elastic collisions and & & & & \\
\hline Rotational excitation $^{\mathrm{d}}$ & & & & 57 \\
\hline Grand total & & & & 959 \\
\hline
\end{tabular}

${ }^{\mathrm{a}}$ For the case $x_{\mathrm{e}}=0$ and $E=1 \mathrm{keV}$

${ }^{\mathrm{b}}$ Dissociation occurs mainly via triplet excitations.

${ }^{\mathrm{c}} \mathrm{He}$ excitation follows the notation in DYL.

dBased on DYL "heating efficiency" in their Table 7. 
is $N=\sum_{i} N_{i}=E \sum_{i} W_{i}^{-1}$ and the average energy per ion pair is

$$
\frac{E}{N}=\left(\sum_{i} W_{i}^{-1}\right)^{-1}
$$

According to Table $1, E / N=36.8 \mathrm{eV}$ per ion pair for $E=1 \mathrm{keV}$ and zero electron fraction.

The data in DYL account for $96 \%$ of the initial electron energy $E=1 \mathrm{keV}$ in Table 1. The small deviation from $100 \%$ is not significant in light of the uncertainties in the calculations. It may be due to the use of the approximate fitting formulae for the parameters $W$, for which DYL quote an accuracy of $5-15 \%$. Another possibility is the omission of triplet states starting near $14 \mathrm{eV}$ that lie above the $a, b$, and $c$ levels, which DYL refer to as missing "pseudo states". In addition, most of the cross sections used in the energy loss process are probably not known to an accuracy of $10 \%$. Approximately $47 \%$ of the energy in Table 1 is accounted for by ionization, $47 \%$ by excitation, and $6 \%$ by direct heating and the collisional de-excitation of $\mathrm{H}_{2}$ rotational levels. Applying this to the $36.8 \mathrm{eV}$ to make an ion pair, elastic scattering and rotational excitation lead to only about $2 \mathrm{eV}$ heating per ion pair in neutral gas. However, as the ionization fraction increases beyond $x_{\mathrm{e}}=10^{-4}$, the direct heating

increases, largely due to Coulomb collisions with ambient electrons. Most cosmic-ray and $\mathrm{X}$-ray heating in molecular regions comes from the reactive ions and excited states. This is exactly the part of the energy loss problem that is not treated by DYL.

\section{The Heating Energy}

In general the heating energy per ion pair $Q$ consists of contributions from collisions with $\mathrm{H}$ and $\mathrm{H}_{2}$,

$$
Q=\frac{x(\mathrm{H}) Q(\mathrm{H})+x\left(\mathrm{H}_{2}\right) Q\left(\mathrm{H}_{2}\right)}{x(\mathrm{H})+x\left(\mathrm{H}_{2}\right)}
$$

\footnotetext{
${ }^{2}$ It is important to note that the DYL considerations do not apply above $x_{\mathrm{e}}=0.1$.
} 
where $x(\mathrm{H})+2 x\left(\mathrm{H}_{2}\right)=1$; the functions $Q(\mathrm{H})$ and $Q\left(\mathrm{H}_{2}\right)$ depend on the abundance of He and especially on the abundance of electrons. The volumetric rate of ion-pair production is $\zeta_{\mathrm{H}} n_{\mathrm{H}}$, where $\zeta_{\mathrm{H}}$ is the ionization rate per $\mathrm{H}$ nucleus and $n_{\mathrm{H}}$ is the volumetric density of $\mathrm{H}$ nuclei. Thus the volumetric heating rate is given by $\Gamma=Q \zeta_{\mathrm{H}} n_{\mathrm{H}}$.

The dominant heating process in atomic regions is elastic scattering. DYL include Coulomb collisions with ambient electrons, but only for the case where the electron abundance relative to total $\mathrm{H}$ nuclei is $<0.1$. For negligible ionization, it is twice that for $\mathrm{H}_{2}$ regions, as can be seen in Table A of the Appendix, the analog of Table 1 for atomic regions. It is included in $Q(\mathrm{H})$, the first term in the numerator of Eq. 2. In Appendix A we show that chemical heating induced by the atomic ions $\mathrm{H}^{+}$and $\mathrm{He}^{+}$reacting with $\mathrm{H}$ is very small. As shown in DYL Figure 8, the dominant excitation product in atomic $\mathrm{H}$ is the $2 p$ level of atomic hydrogen. The resultant Ly- $\alpha$ photons will either escape or be absorbed by and heat the dust, and not the gas.

The heating in molecular regions is the sum of the effects from elastic collisions plus rotational excitation $\left(Q_{\mathrm{el} / \mathrm{rot}}\right)$, excitation of $\mathrm{H}_{2}$ vibrational levels $\left(Q_{\mathrm{vib}}\right)$, dissociation of $\mathrm{H}_{2}$ $\left(Q_{\text {diss }}\right)$, and chemical heating $\left(Q_{\text {chem }}\right)$ :

$$
Q\left(\mathrm{H}_{2}\right)=Q_{\mathrm{el} / \mathrm{rot}}+Q_{\mathrm{vib}}+Q_{\mathrm{diss}}+Q_{\mathrm{chem}}
$$

In the following paragraphs we discuss the first three contributions to $Q\left(\mathrm{H}_{2}\right)$, and then focus on $Q_{\text {chem }}$ in the next section.

\subsection{Elastic Scattering and Rotational Excitation}

The critical densities for the rotational levels of $\mathrm{H}_{2}$ depend on the transition and also

on the $\mathrm{H} / \mathrm{H}_{2}$ ratio and the temperature (e.g., Le Bourlot et al. 1999). In many situations the lowest $\mathrm{S}(0)$ and $\mathrm{S}(1)$ rotational levels are collisionally de-excited and most of the rotational 
excitation goes into heating. GL73 estimated that a density $n_{\mathrm{H}}=1000 \mathrm{~cm}^{-3}$ would satisfy this condition on density, independent of temperature. We use DYL for the combined heating due to elastic scattering and rotational excitation,

$$
Q_{\mathrm{el} / \mathrm{rot}}\left(\mathrm{H}_{2}, \mathrm{e}\right) \equiv Q_{\mathrm{el}}\left(\mathrm{H}_{2}, \mathrm{e}\right)+Q_{\mathrm{rot}}\left(\mathrm{H}_{2}, \mathrm{e}\right)
$$

This quantity is then expressed in terms of a "heating efficiency" $\eta$ for a $\mathrm{H}_{2}$, He gas mixture,

$$
Q_{\mathrm{el} / \mathrm{rot}}\left(\mathrm{H}_{2}, \mathrm{e}\right)=\eta\left(\mathrm{H}_{2}, \mathrm{e}\right) W\left(\mathrm{H}_{2}, \mathrm{e}\right)
$$

where $W\left(\mathrm{H}_{2}, \mathrm{e}\right)$ is the average energy to make an ion pair. Both $\zeta_{\mathrm{H}}$ and the heating function $Q$ are defined in terms of ion pairs. For the $\mathrm{H}$, He gas, there is of course no rotational heating, and the heating is expressed in a similar form where the heating efficiency $\eta(\mathrm{H}, \mathrm{e})$ now describes only elastic collisions,

$$
Q_{\mathrm{el}}(\mathrm{H}, \mathrm{e})=\eta(\mathrm{H}, \mathrm{e}) W(\mathrm{H}, \mathrm{e})
$$

However, $W(\mathrm{H}, \mathrm{e}) \approx W\left(\mathrm{H}_{2}, \mathrm{e}\right)$ to an accuracy of $\approx 5 \%$ for electron energies greater than several hundred eV, and the first term of Eq. 3 becomes,

$$
Q_{\mathrm{el}}(\mathrm{H}, \mathrm{e})+Q_{\mathrm{el} / \mathrm{rot}}\left(\mathrm{H}_{2}, \mathrm{e}\right) \approx \frac{\left[x(\mathrm{H}) \eta(\mathrm{H}, \mathrm{e})+x\left(\mathrm{H}_{2}\right) \eta\left(\mathrm{H}_{2}, \mathrm{e}\right)\right]}{x(\mathrm{H})+x\left(\mathrm{H}_{2}\right)} W\left(\mathrm{H}_{2}, \mathrm{e}\right)
$$

with $W\left(\mathrm{H}_{2}, \mathrm{e}\right) \approx 37 \mathrm{eV}$ asymptotically for $E \geq 500 \mathrm{eV}$.

The heating efficiencies $\eta$ are expressed by the fitting formulae, DYL Eq. (14),

$$
\eta=1-\frac{1-\eta_{0}}{1+C x_{\mathrm{e}}^{\alpha}}
$$

The two sets of fit parameters are given at the bottom of DYL Table 7 ("Two-Gas Mixtures") as a function of electron energy $E$. For example, the $E=1 \mathrm{keV}$ values are,

$$
\eta(\mathrm{H}, \mathrm{e})_{0}=0.117, \quad \alpha=0.678, \quad C=7.95,
$$


for atomic regions, and

$$
\eta\left(\mathrm{H}_{2}, \mathrm{e}\right)_{0}=0.055, \quad \alpha=0.366, \quad C=2.17
$$

for molecular regions. These values might be appropriate for the $\mathrm{keV}$ X-rays emitted by young stellar objects. These fits have only a modest accuracy $\sim 10-15 \%$.

According to DYL (see Table 1), $Q_{\mathrm{el} / \mathrm{rot}}$ accounts for only $57 \mathrm{eV}$, or $\sim 6 \%$ of the energy of the 1-keV incident electron. The rest of the energy is almost equally shared by ionization and excitation of He and $\mathrm{H}_{2}$. Thus, the DYL heating efficiency for molecular regions is only a small part of the total. With $W \sim 37 \mathrm{eV}$ to make an ion pair, elastic scattering and rotational excitation lead to $Q_{\mathrm{el} / \mathrm{rot}}=2.1 \mathrm{eV}$ heating per ion pair in neutral $\mathrm{H}_{2}$, He gas. This is half of the direct heating in an atomic $\mathrm{H}, \mathrm{He}, \sim 4.3 \mathrm{eV}$ under the same conditions.

\subsection{Dissociation Heating}

The most important pathway to dissociation is collisional excitation of $\mathrm{H}_{2}$ triplet states that start at $11.9 \mathrm{eV}(a$ and $c), 13.4 \mathrm{eV}(e)$ and possibly above. These levels decay into continuum states $b$ of the triplet repulsive potential and have essentially unit probability for dissociation. Unlike the Lyman and Werner transitions to the singlet $B$ and $C$ levels, these spin-flip transitions are forbidden for photon excitation from the singlet ground state $X$. Table 1 indicates that for $E=1 \mathrm{keV}$ and $x_{\mathrm{e}}=0$, there are 10.8 dissociations per $\mathrm{keV}$. Adopting the DYL estimate of $5.4 \mathrm{eV}$ per dissociation, we obtain $58 \mathrm{eV}$ heating per $\mathrm{keV}$, or $2.14 \mathrm{eV}$ per ion pair for a neutral $\mathrm{H}_{2}$, He mixture 3. More generally we use DYL Table 5 for

\footnotetext{
${ }^{3}$ This number does not include the heating from dissociation following collisionalexcitation of the $B$ and $C$ states, which is much smaller, only $\sim 0.6 \mathrm{eV}$ per keV.
} 
the dissociation heating in Eq. 3,

$$
Q_{\mathrm{diss}}=\frac{x\left(\mathrm{H}_{2}\right)}{x(\mathrm{H})+x\left(\mathrm{H}_{2}\right)} \frac{D_{0}}{1+C x_{\mathrm{e}}^{\alpha}}
$$

where $D_{0}=2.14 \mathrm{eV}, \alpha=0.574$ and $C=22.0$.

\subsection{Vibrational Heating}

Fast electrons can excite the vibrational levels of $\mathrm{H}_{2}$ by direct collisions or by fluorescent de-excitation of electronically excited $B$ and $C$ levels. The excitation energy goes into heating if the densities are high enough for the levels to be collisionally de-excited. Recent calculations of the collisional de-excitation rate coefficients (e.g. Wrathmall et al. 2007 for H; Lee et al. 2008 for $\mathrm{H}_{2}$; Balakrishnan et al. 1999 for He) replace earlier work of Tiné et al. (1997) and Le Bourlot et al. (1999). The coefficients are generally much larger for collisions with atomic $\mathrm{H}$ than for $\mathrm{H}_{2}$ and He. They are very small below $100 \mathrm{~K}$ and increase rapidly above this temperature. Thus one can expect little quenching of vibrational levels in the cool and moderately dense regions of molecular clouds. By contrast, in the surface layers of the inner regions of protoplanetary disks, where $n_{\mathrm{H}} \sim 10^{10} \mathrm{~cm}^{-3}$ and $T \sim 1000 \mathrm{~K}$, the densities of both $\mathrm{H}$ and $\mathrm{H}_{2}$ are large enough to collisionally quench vibrationally-excited $\mathrm{H}_{2}$ molecules and provide significant heating. In less dense molecular regions, atomic $\mathrm{H}$ collisions may be effective in collisionally de-exciting $\mathrm{H}_{2}$, but this depends on the temperature and the $\mathrm{H} / \mathrm{H}_{2}$ ratio as well as the total density $n_{\mathrm{H}}$.

In order to obtain the heating from direct collisional excitation in dense $\mathrm{H}_{2}$ regions, we use DYL Table 5 for $\mathrm{H}_{2}$, He mixtures which gives the following fit for the energy to excite the $v=1,2$ levels,

$$
\epsilon_{v}=W_{v}\left(1+C_{v} x_{\mathrm{e}}^{\alpha_{v}}\right)
$$

The parameters for $E=1 \mathrm{keV}$ are given in Table 2 for $E \geq 500 \mathrm{eV}$, with $W_{v}$ and $\Delta E_{v}$ (the 
vibrational excitation energies) in $\mathrm{eV}$.

Because $1000 / \epsilon_{v}$ is the number of excitations per keV of electron energy, the high-density direct vibrational heating per keV of incident electron energy is,

$$
\left(\frac{1000}{\epsilon_{1}} \Delta E_{1}+\frac{1000}{\epsilon_{2}} \Delta E_{2}\right) \mathrm{eV} .
$$

The vibrational heating per ion pair is obtained by dividing this expression by the number of ion pairs per $\mathrm{keV}$ (27.2 for $E \geq 500 \mathrm{eV}$ ), with the result,

$$
Q_{\mathrm{dir} / \mathrm{vib}} \approx \frac{x\left(\mathrm{H}_{2}\right)}{x(\mathrm{H})+x\left(\mathrm{H}_{2}\right)} 19.0 \mathrm{eV}\left[\frac{1}{\epsilon_{1}}+\frac{2}{\epsilon_{2}}\right]
$$

In a pure $\mathrm{H}_{2}$ region with negligible ionization, the vibrational heating per ion pair is $2.8 \mathrm{eV}$ for $E \geq 500 \mathrm{eV}$, with $v=2$ contributing about $15 \%$.

Excited vibrational levels are also produced when collisionally excited $B$ and $C$ states decay with the emission of fluorescent photons in the 1500-1600 $\AA$ range, with a maximum near $1575 \AA$. We can calculate the high-density heating that follows the collisional de-excitation of the vibrational levels using the above method for direct collisional excitation based on Eq. 12, The necessary parameters again come from DYL Table 5 for $\mathrm{H}_{2}$, He mixtures.

Table 2. Direct Vibrational Parameters ${ }^{\mathrm{a}}$

\begin{tabular}{ccccc}
\hline \hline$v$ & $W_{v}(\mathrm{eV})$ & $C_{v}$ & $\alpha_{v}$ & $\Delta E_{v}(\mathrm{eV})$ \\
\hline & & & & \\
1 & 7.81 & 23,500 & 0.955 & 0.516 \\
2 & 109 & 10,700 & 0.907 & 1.032 \\
\hline
\end{tabular}

${ }^{\mathrm{a}} E=1 \mathrm{keV}$ 
With roughly $4 \mathrm{eV}$ in vibrational excitation energy and 27.2 ion pairs per $\mathrm{keV}$, the high-density $B, C$ vibrational heating per keV of incident electron energy is,

$$
\frac{1000 / \epsilon_{B}+1000 / \epsilon_{C}}{27.2} 4.0 \mathrm{eV}=147 \mathrm{eV}\left[\frac{1}{\epsilon_{B}}+\frac{1}{\epsilon_{C}}\right] .
$$

The $B, C$ vibrational heating per ion pair is then,

$$
Q_{B C / \mathrm{vib}}=\frac{x\left(\mathrm{H}_{2}\right)}{x(\mathrm{H})+x\left(\mathrm{H}_{2}\right)} 147 \mathrm{eV}\left[\frac{1}{\epsilon_{B}}+\frac{1}{\epsilon_{C}}\right] .
$$

If we substitute the values in Table 3 for $x_{\mathrm{e}}=0$, the result is $2.4 \mathrm{eV}$ per ion pair for a neutral $\mathrm{H}_{2}$, He gas, a small but not negligible contribution to the high-density heating.

As discussed earlier, both Eqs. 13 and 14 hold only if the density well exceeds the critical density $n_{\mathrm{cr}}$ for the de-excitation of the vibrational transitions of the $\mathrm{H}_{2}$ ground level. They therefore should be multiplied by an appropriate factor $\Theta_{\text {vib }}$, that depends on the ratio $n_{\mathrm{cr}} / n_{\mathrm{H}}$, such that $\Theta_{\mathrm{vib}}=0$ for small $n_{\mathrm{cr}} / n_{\mathrm{H}}$ and $\Theta_{\mathrm{vib}}=1$ for large $n_{\mathrm{cr}} / n_{\mathrm{H}}$. Their contribution to the total heating in Eq. 3 is now,

$$
Q_{\mathrm{vib}}=\Theta_{\mathrm{vib}}\left(Q_{\mathrm{dir} / \mathrm{vib}}+Q_{B C / \mathrm{vib}}\right)
$$

The $B$ and $C$ levels have a $\sim 15 \%$ probability to dissociate (e.g., Abgrall et al. 1997), with a yield of approximately $0.25 \mathrm{eV}$ in typical interstellar conditions (Tielens 2000). The

Table 3. $B, C$ Vibrational Parameters ${ }^{\mathrm{a}}$

\begin{tabular}{lrrr}
\hline \hline & & & \\
Level & $W_{v}(\mathrm{eV})$ & $C_{v}$ & $\alpha_{v}$ \\
\hline & & & \\
$B$ & 117 & 7.09 & 0.779 \\
$C$ & 132 & 6.88 & 0.802 \\
\hline & & & \\
${ }^{\mathrm{a}} E=1 \mathrm{keV}$
\end{tabular}


small branching ratio for dissociation and the small energy yield mean that the heating per ion pair following dissociation is very small, e.g., $0.02 \mathrm{eV}$ for $E=1 \mathrm{keV}$, and thus negligible.

\section{Chemical Heating}

Chemical heating derives mainly from reactions instigated by the primary cosmic-ray and X-ray ions, $\mathrm{H}_{2}^{+}, \mathrm{H}^{+}$, and $\mathrm{He}^{+}$, with neutral species and electrons. It was first considered in the context of EUV heating of Jupiter's upper atmosphere (Henry \& McElroy 1969) and then for cosmic ray ionization of interstellar molecular clouds (GL73). These authors focused on $\mathrm{H}_{2}^{+}$, its transformation into $\mathrm{H}_{3}^{+}$, and the destruction of $\mathrm{H}_{3}^{+}$by dissociative recombination. In dense molecular regions, however, where $x_{\mathrm{e}} \ll x\left(\mathrm{H}_{2}\right)$, dissociative recombination has to compete against ionic reactions with neutral species. Rather than treat all of the ionneutral reactions that can be traced to the primary ions, $\mathrm{H}_{2}^{+}, \mathrm{H}^{+}$, and $\mathrm{He}^{+}$, we focus on only the potentially most abundant neutrals: $\mathrm{CO}, \mathrm{H}_{2} \mathrm{O}$, and $\mathrm{O}$. Although this treatment is not completely general, it should suffice to demonstrate the nature and magnitude of chemical heating in molecular regions. The chemical heating will be expressed in terms of its values for the three main initiating ions in $\mathrm{H}_{2}$ regions,

$$
Q_{\text {chem }}\left(\mathrm{H}_{2}\right)=Q_{\text {chem }}\left(\mathrm{H}_{2}^{+}, \mathrm{H}_{2}\right)+Q_{\text {chem }}\left(\mathrm{H}^{+}, \mathrm{H}_{2}\right)+Q_{\text {chem }}\left(\mathrm{He}^{+}, \mathrm{H}_{2}\right) .
$$

According to the discussion of Table 1 in Section 2, each of these terms includes a weighting factor equal to the fraction for each ion of the total, $F\left(\mathrm{H}_{2}^{+}\right)=0.88, F\left(\mathrm{H}^{+}\right)=0.04$, and $F\left(\mathrm{He}^{+}\right)=0.08$, ignoring the tiny fraction of $\mathrm{He}^{2+}$. In Appendix A we show that chemical heating in atomic regions is negligible. 


\subsection{Chemical Heating of $\mathrm{H}_{2}$}

As discussed in Section 2, the most abundant ion generated by cosmic-rays and X-rays in $\mathrm{H}_{2}$ regions is $\mathrm{H}_{2}^{+}$. We first consider its main destruction routes; $\mathrm{H}^{+}$and $\mathrm{He}^{+}$ions will be analyzed later in this section. $\mathrm{H}_{2}^{+}$is mainly destroyed by dissociative recombination and by proton transfer with $\mathrm{H}_{2}$,

$$
\mathrm{H}_{2}^{+}+\mathrm{e} \rightarrow \mathrm{H}+\mathrm{H}, \quad \mathrm{H}_{2}^{+}+\mathrm{H}_{2} \rightarrow \mathrm{H}_{3}^{+}+\mathrm{H}
$$

with rate coefficients, $\beta=2.0 \times 10^{-7} T^{-1 / 2} \mathrm{~cm}^{3} \mathrm{~s}^{-1}$ (a rough fit to Schneider et al. 1994) and $k=2 \times 10^{-9} \mathrm{~cm}^{3} \mathrm{~s}^{-1}$ (Theard \& Huntress 1974). The probability for $\mathrm{H}_{3}^{+}$production is,

$$
P\left(\mathrm{H}_{2}^{+}, \mathrm{H}_{3}^{+}\right)=\frac{k x\left(\mathrm{H}_{2}\right)}{k x\left(\mathrm{H}_{2}\right)+\beta x_{\mathrm{e}}}=\frac{x\left(\mathrm{H}_{2}\right)}{x\left(\mathrm{H}_{2}\right)+100 T^{-1 / 2} x_{\mathrm{e}}},
$$

so that if,

$$
x_{\mathrm{e}}<0.1 \frac{k}{\beta} x\left(\mathrm{H}_{2}\right)=0.01\left(\frac{T}{100 \mathrm{~K}}\right)^{1 / 2} x\left(\mathrm{H}_{2}\right),
$$

the rate of dissociative recombination is less than $10 \%$ that for $\mathrm{H}_{2}$ destruction. For regions with considerable $\mathrm{H}_{2}$, this condition is well satisfied, $P\left(\mathrm{H}_{2}^{+}, \mathrm{H}_{3}^{+}\right) \approx 1$, and most of the $\mathrm{H}_{2}^{+}$ ions are transformed into $\mathrm{H}_{3}^{+}$.

The destruction pathways for $\mathrm{H}_{3}^{+}$are first, dissociative recombination with two branches,

$$
\mathrm{H}_{3}^{+}+\mathrm{e} \rightarrow \mathrm{H}_{2}+\mathrm{H}(25 \%) \text { and } \mathrm{H}+\mathrm{H}+\mathrm{H}(75 \%)
$$

and total rate coefficient $\beta^{\prime}=4.5 \times 10^{-6} T^{-0.65} \mathrm{~cm}^{3} \mathrm{~s}^{-1}$ (Sundström et al. 1994), and second, proton-transfer reactions such as,

$$
\begin{array}{rll}
\mathrm{H}_{3}^{+}+\mathrm{CO} & \rightarrow \mathrm{HCO}^{+}+\mathrm{H}_{2} & k_{1}=1.6 \times 10^{-9} \mathrm{~cm}^{3} \mathrm{~s}^{-1} \\
\mathrm{H}_{3}^{+}+\mathrm{H}_{2} \mathrm{O} & \rightarrow \mathrm{H}_{3} \mathrm{O}^{+}+\mathrm{H} & k_{2}=5.3 \times 10^{-9} \mathrm{~cm}^{3} \mathrm{~s}^{-1} \\
\mathrm{H}_{3}^{+}+\mathrm{O} & \rightarrow \mathrm{OH}^{+}+\mathrm{H}_{2} & k_{3}=0.8 \times 10^{-9} \mathrm{~cm}^{3} \mathrm{~s}^{-1}
\end{array}
$$


Dissociative recombination, Eq. 20, was considered by GL73. It is competitive with the reactions in Eq. 21 where the electron fraction is relatively large. Denoting the abundance of the neutral reactants in Eq. 21 by $x_{i}$ with $i=1,2,3$ for $\mathrm{CO}, \mathrm{H}_{2} \mathrm{O}$, and $\mathrm{O}$, respectively, we introduce branching ratios for the main reaction pathways of $\mathrm{H}_{3}^{+}$,

$$
B_{\mathrm{e}}=\frac{\beta^{\prime} x_{\mathrm{e}}}{\sum_{i} k_{i} x_{i}+\beta^{\prime} x_{\mathrm{e}}} \quad B_{i}=\frac{k_{i} x_{i}}{\sum_{i} k_{i} x_{i}+\beta^{\prime} x_{\mathrm{e}}} \quad(i=1,2,3) .
$$

In order for dissociative recombination to compete with ionic reactions at the $10 \%$ level, the electron fraction must satisfy the condition,

$$
x_{\mathrm{e}}>0.1 \frac{\Sigma_{i} k_{i} x_{i}}{\beta^{\prime}}=2.22 \times 10^{-9} T^{0.65}\left(\Sigma_{i} k_{i} x_{i}\right)_{-13}
$$

where $\left(\sum_{i} k_{i} x_{i}\right)_{-13}=\left(\Sigma_{i} k_{i} x_{i} / 10^{-13} \mathrm{~cm}^{3} \mathrm{~s}^{-1}\right)$ measures the reactivity of $\mathrm{H}_{3}^{+}$with abundant neutrals in units determined by neutral abundances of order $10^{-4}$ and ionic reactions with rate coefficients of order $10^{-9} \mathrm{~cm}^{3} \mathrm{~s}^{-1}$. Even in cold molecular regions, $(T \sim 10 \mathrm{~K})$ dissociative recombination can still play a role in the presence of neutral reactions. Eq. 23 for $\mathrm{H}_{3}^{+}$is much less restrictive than Eq. 19 for $\mathrm{H}_{2}^{+}$because of the reduced abundance of heavy elements compared to hydrogen.

The first reaction in Eq. 21 with $\mathrm{CO}$ is simple to treat because the product $\mathrm{HCO}^{+}$ is mainly destroyed in one step by dissociative recombination back to $\mathrm{CO}+\mathrm{H}$, whereas dissociative recombination of $\mathrm{H}_{3} \mathrm{O}^{+}$in the second reaction has three branches, $\mathrm{H}_{2} \mathrm{O}+\mathrm{H}$, $\mathrm{OH}+\mathrm{H}_{2}$, and $\mathrm{OH}+2 \mathrm{H}$. The situation for the reaction with $\mathrm{O}$ is similar because $\mathrm{OH}^{+}$in the third equation is quickly transformed into $\mathrm{H}_{3} \mathrm{O}^{+}$by hydrogenation reactions with $\mathrm{H}_{2}$. In this sequence, we can ignore dissociative recombination of $\mathrm{OH}^{+}$and $\mathrm{H}_{2} \mathrm{O}^{+}$because of conditions similar to Eq. 19.

Keeping in mind that the probability for the conversion of $\mathrm{H}_{2}^{+}$into $\mathrm{H}_{3}^{+}, P\left(\mathrm{H}_{2}^{+}, \mathrm{H}_{3}^{+}\right)$, is essentially unity according to Eqs. 18 and 19, the chemical energy released by the creation of an $\mathrm{H}_{2}^{+}$ion can be obtained by consolidating the reactions in Eq. 17 and Eq. 21 into the 
equivalent reactions,

$$
\begin{gathered}
\mathrm{e}+\mathrm{H}_{2}^{+} \rightarrow \mathrm{H}+\mathrm{H} \\
\mathrm{e}+\mathrm{H}_{2}^{+}+\mathrm{H}_{2} \mathrm{O}+\mathrm{H}_{2} \rightarrow \mathrm{H}_{2} \mathrm{O}+\mathrm{H}_{2}+2 \mathrm{H}, \quad \mathrm{OH}+2 \mathrm{H}_{2}+\mathrm{H}, \quad \mathrm{OH}+\mathrm{H}_{2}+3 \mathrm{H} \\
\mathrm{e}+\mathrm{H}_{2}^{+}+\mathrm{O}+2 \mathrm{H}_{2} \rightarrow \mathrm{H}_{2} \mathrm{O}+4 \mathrm{H}, \quad \mathrm{OH}+\mathrm{H}_{2}+3 \mathrm{H}, \mathrm{OH}+5 \mathrm{H}
\end{gathered}
$$

where $\bar{q}_{1}=11.1 \mathrm{eV}, \bar{q}_{2}=7.8 \mathrm{eV}$, and $\bar{q}_{3}=5.7 \mathrm{eV}$ are the net energy yields for each of the three consolidated reactions of $\mathrm{H}_{2}^{+}$, Eqs. 24, 25] and 26. The $\bar{q}$ are averages over the outcomes generated by the three branches in the dissociative recombination of $\mathrm{H}_{3} \mathrm{O}^{+}$,

$$
\mathrm{H}_{3} \mathrm{O}^{+}+\mathrm{e} \rightarrow \mathrm{H}_{2} \mathrm{O}+\mathrm{H}(25 \%) ; \quad \mathrm{OH}+\mathrm{H}_{2}(15 \%) ; \quad \mathrm{OH}+2 \mathrm{H}(60 \%) .
$$

For example, Eq. 24 results from adding the full sequence of reactions that are involved when $\mathrm{H}_{3}^{+}$reacts with $\mathrm{CO}$ :

$$
\begin{aligned}
\mathrm{H}_{2}^{+}+\mathrm{H}_{2} & \rightarrow \mathrm{H}_{3}^{+}+\mathrm{H} \\
\mathrm{H}_{3}^{+}+\mathrm{CO} & \rightarrow \mathrm{HCO}^{+}+\mathrm{H}_{2} \\
\mathrm{e}+\mathrm{H}_{2}^{+} & \rightarrow \mathrm{CO}+\mathrm{H} .
\end{aligned}
$$

The chemical heating due to the reactions of the $\mathrm{H}_{2}^{+}$ion is then the rate at which that ion is produced per unit volume multiplied by the probability that it is transformed into an $\mathrm{H}_{3}^{+}$ion, (Eq. 18), times the heating averaged over the three branches of the latter's reactions and including the heating from the dissociative recombination of $\mathrm{H}_{3}^{+}$, all multiplied by the $\mathrm{H}_{2}$ fraction. In terms of the notation of Eq. 16, the chemical heating per ion pair stemming from the production of the $\mathrm{H}_{2}^{+}$ion is,

$$
Q_{\text {chem }}\left(\mathrm{H}_{2}^{+}\right)=\frac{x\left(\mathrm{H}_{2}\right)}{x(\mathrm{H})+x\left(\mathrm{H}_{2}\right)} F\left(\mathrm{H}_{2}^{+}\right) P\left(\mathrm{H}_{2}^{+}, \mathrm{H}_{3}^{+}\right)\left[\Sigma_{i} B_{i}\left(\mathrm{H}_{2}^{+}\right) \bar{q}_{i}\left(\mathrm{H}_{2}^{+}\right)+B_{\mathrm{e}} \bar{q}_{\mathrm{e}}\left(\mathrm{H}_{2}^{+}\right)\right] .
$$

The heating energies $\bar{q}_{i}$ are given following Eq. 26, and the branchings are defined in Eq. 22. $q_{\mathrm{e}}\left(\mathrm{H}_{2}^{+}\right)=7.6 \mathrm{eV}$. For the case where ion-molecule destruction prevails over dissociative recombination, we can estimate the value of the chemical heating for the case where the 
abundances for $\mathrm{CO}, \mathrm{H}_{2} \mathrm{O}$, and $\mathrm{O}$ all equal $10^{-4}$. The result is $\Sigma_{i} B_{i}\left(\mathrm{H}_{2}^{+}\right) q_{i}\left(\mathrm{H}_{2}^{+}\right)=8.4 \mathrm{eV}$, but this must still be multiplied by $F\left(\mathrm{H}_{2}^{+}\right)=0.88$ to yield $Q_{\text {chem }}\left(\mathrm{H}_{2}^{+}\right)=7.2 \mathrm{eV}$.

Examination of Eqs. 25] and 26 reveals that the products of the ion-molecule reactions for $\mathrm{H}_{2} \mathrm{O}$ and $\mathrm{O}$ involve the radicals $\mathrm{OH}$ and $\mathrm{H}$ whose further reaction can lead to more chemical heating. For example, $\mathrm{OH}$ can be converted to $\mathrm{H}_{2} \mathrm{O}$ by the exothermic reaction,

$$
\mathrm{OH}+\mathrm{H}_{2} \rightarrow \mathrm{H}_{2} \mathrm{O}+\mathrm{H}
$$

and the $\mathrm{H}$ atoms can make $\mathrm{H}_{2}$ by formation on dust grain surfaces "gr",

$$
\mathrm{H}+\mathrm{H}+\mathrm{gr} \rightarrow \mathrm{H}_{2}+\mathrm{gr}^{\prime}
$$

The energy yield of the $\mathrm{OH}$ reaction is $0.65 \mathrm{eV}$. When this is added to the energy yields of Eqs. 25 and 26, the chemical heating produced in these reactions is increased slightly, $\bar{q}_{2}=$ $8.4 \mathrm{eV}$ and $\bar{q}_{3}=6.4 \mathrm{eV}$. The previous estimate of $\Sigma_{i} B_{i} \bar{q}_{i}$ for the case of equal abundances for $\mathrm{CO}, \mathrm{H}_{2} \mathrm{O}$, and $\mathrm{O}\left(\right.$ all $\left.10^{-4}\right)$ is increased from $8.4 \mathrm{eV}$ to $8.8 \mathrm{eV}$, and $Q_{\text {chem }}\left(\mathrm{H}_{2}^{+}\right)=7.7 \mathrm{eV}$.

The heating from Eq. 30 depends on how much if any of the energy release goes into kinetic energy of the newly formed molecule compared to internal excitation and excitation of the birth grain. Theoretical estimates and guesses in the literature range from 0.5 to $3.0 \mathrm{eV}$, but laboratory experiments (e.g., Roser et al. 2003) suggest that the $\mathrm{H}_{2}$ molecule thermally accommodates to the temperature of the grain before making its final escape, at least as far as kinetic energy is concerned. In a recent experiment, Lemaire et al. (2010) present evidence that $\sim 30 \%$ of newly formed $\mathrm{H}_{2}$ molecules are vibrationally excited for dust temperatures as warm as $70 \mathrm{~K}$. Assuming that the results of Lemaire et al. applies to molecular regions where the density is large enough for vibrationally excited $\mathrm{H}_{2}$ molecules to be collisionally de-excited, the chemical heating for the $\mathrm{H}_{2} \mathrm{O}$ and $\mathrm{O}$ channels would be increased by $1.5 \mathrm{eV}$. This would then lead to an increase in the heating function by about $1 \mathrm{eV}$ to $Q_{\text {chem }}\left(\mathrm{H}_{2}^{+}\right) \sim 9 \mathrm{eV}$. In the numerical estimates below, we ignore this contribution pending the resolution of this long standing issue regarding $\mathrm{H}_{2}$ formation on grains. 


\subsection{Chemical Heating of $\mathrm{H}^{+}$and $\mathrm{He}^{+}$}

In principle, the chemical heating of $\mathrm{H}^{+}$and $\mathrm{He}^{+}$ions can be treated in a similar way as $\mathrm{H}_{2}^{+}$, but the outcomes depend on the chemical composition of the molecular region in question. Thus $\mathrm{H}^{+}$does not react with $\mathrm{CO}$, the most abundant and stable heavy molecule, and its fast charge-exchange with $\mathrm{O}$ is closely balanced by the reverse reaction. It does react with $\mathrm{H}_{2} \mathrm{O}$, which can be very abundant under certain circumstances, and with many organic species that usually have small abundances. In a cold molecular cloud, where $\mathrm{H}_{2} \mathrm{O}$ may be expected to be frozen out on grains, this route will be shut down. On the other hand, in a dense warm region like those observed in the inner regions of protoplanetary disks, the primary destruction pathway for $\mathrm{H}^{+}$is

$$
\mathrm{H}^{+}+\mathrm{H}_{2} \mathrm{O} \rightarrow \mathrm{H}_{2} \mathrm{O}^{+}+\mathrm{H}
$$

followed by hydrogenation to $\mathrm{H}_{3} \mathrm{O}^{+}$,

$$
\mathrm{H}_{2} \mathrm{O}^{+}+\mathrm{H}_{2} \rightarrow \mathrm{H}_{3} \mathrm{O}^{+}+\mathrm{H}
$$

and destruction of $\mathrm{H}_{3} \mathrm{O}^{+}$by dissociative recombination, according to Eq. 27. The energy balance equation is similar to Eq. 25 for $\mathrm{H}_{2}^{+}$reacting with $\mathrm{H}_{2} \mathrm{O}$. The only difference is that $\mathrm{H}_{2}^{+}$is replaced by $\mathrm{H}^{+}$on the left side and $\mathrm{H}_{2}$ is replaced by $\mathrm{H}$ on the right side. The net

change in exothermic reaction yield (and in the maximum heating) is just the difference between the ionization potentials of $\mathrm{H}_{2}$ and $\mathrm{H}$, or $1.83 \mathrm{eV}$. Thus the chemical heating arising from $\mathrm{H}^{+}$analogous to Eq. 28 is,

$$
Q_{\text {chem }}\left(\mathrm{H}^{+}\right)=\frac{x\left(\mathrm{H}_{2}\right)}{x(\mathrm{H})+x\left(\mathrm{H}_{2}\right)} F\left(\mathrm{H}^{+}\right) B_{2}\left(\mathrm{H}^{+}\right) \bar{q}_{i}\left(\mathrm{H}^{+}\right)
$$

where $F\left(\mathrm{H}^{+}\right)$is the fraction of $\mathrm{H}^{+}$ions $(0.040$ from Table 1$), B_{2}\left(\mathrm{H}^{+}\right) \simeq 1$ is the branching ratio for $\mathrm{H}^{+}$to react with $\mathrm{H}_{2} \mathrm{O}$, and $\bar{q}_{2}\left(\mathrm{H}^{+}\right)=\bar{q}_{2}\left(\mathrm{H}_{2}^{+}\right)-1.83 \mathrm{eV}$. In principle, $B_{2}\left(\mathrm{H}^{+}\right)$is given by an expression similar to Eq. 22 with $\beta^{\prime}$, the dissociative recombination rate coefficient 
for $\mathrm{H}_{3}^{+}$, replaced by the radiative recombination rate for $\mathrm{H}^{+}$. Because the latter is smaller by many orders of magnitude, destruction of $\mathrm{H}^{+}$by radiative recombination can be ignored. For a completely molecular region, $F\left(\mathrm{H}^{+}\right)=0.04, B_{2} \sim 1$, and $\bar{q}_{2}\left(\mathrm{H}^{+}\right)=6.9 \mathrm{eV}$, resulting in $Q_{\text {chem }}\left(\mathrm{H}^{+}\right)=0.28 \mathrm{eV}$. This estimate includes the energy that can be recovered from the $\mathrm{OH}$ radical but not from the conversion of product $\mathrm{H}$ atoms to form $\mathrm{H}_{2}$ on grains. It is much smaller than the heating per ion pair for $\mathrm{H}_{2}^{+}$because $\mathrm{H}^{+}$is less energetic and because only $4 \%$ of the ion pairs involve $\mathrm{H}^{+}$. This estimate is also sensitive to the physical conditions because the $\mathrm{H}_{2} \mathrm{O}$ abundance can vary, especially due to freeze out on grains, and because reactions with other species may occur such as charge transfer with neutral heavy atoms.

The heating by the $\mathrm{He}^{+}$ions is more complicated because it reacts with many molecules including $\mathrm{H}_{2}$. Here we consider the dominant reaction to be,

$$
\mathrm{He}^{+}+\mathrm{CO} \rightarrow \mathrm{C}^{+}+\mathrm{O}+\mathrm{He},
$$

followed by,

$$
\mathrm{C}^{+}+\mathrm{H}_{2} \mathrm{O} \rightarrow \mathrm{HCO}^{+}+\mathrm{H},
$$

and then by dissociative recombination of $\mathrm{HCO}^{+}$. A similar analysis to that followed above for $\mathrm{H}_{2}^{+}$gives an energy budget equation,

$$
\mathrm{e}+\mathrm{He}^{+}+\mathrm{H}_{2} \mathrm{O} \rightarrow \mathrm{O}+2 \mathrm{H}+\mathrm{He},
$$

with an energy yield of $15.0 \mathrm{eV}$. Including the reactions of the remaining $\mathrm{O}$ atom gives another $0.6 \mathrm{eV}$, or $q_{1}\left(\mathrm{He}^{+}\right)=15.6 \mathrm{eV}$. On this simplified basis, the heating due to $\mathrm{He}^{+}$ions is

$$
Q_{\text {chem }}\left(\mathrm{He}^{+}\right)=\frac{x\left(\mathrm{H}_{2}\right)}{x(\mathrm{H})+x\left(\mathrm{H}_{2}\right)} F\left(\mathrm{He}^{+}\right) B_{1}\left(\mathrm{He}^{+}\right) q_{1}\left(\mathrm{He}^{+}\right) .
$$

Using $F\left(\mathrm{He}^{+}\right)=0.80, B_{1}\left(\mathrm{He}^{+}\right)=1$ (radiative recombination is again unimportant in molecular regions), $Q_{\text {chem }}\left(\mathrm{He}^{+}\right)=1.25 \mathrm{eV}$. This may be a slight overestimate because other reactions of $\mathrm{He}^{+}$have been neglected, e.g., with water. 


\subsection{Summary of Chemical Heating}

To summarize this treatment of chemical heating, we have considered the energy that is available for heating due to the reactions of $\mathrm{H}_{2}^{+}, \mathrm{H}^{+}$, and $\mathrm{He}^{+}$ions, which account respectively for $88 \%, 4 \%$, and $8 \%$ of the $\sim 27$ ion pairs per keV produced by high energy electrons $(E>500 \mathrm{eV})$. The $\mathrm{H}_{2}^{+}$ion accounts for $7.7 \mathrm{eV}$ per ion pair, which is most of the chemical heating $(84 \%)$. This is very close to the heating from the dissociative recombination of $\mathrm{H}_{3}^{+}$, $\simeq 7.6 \mathrm{eV}$ (GL73). Even the total chemical heating, $9.2 \mathrm{eV}$ per ion pair, is insensitive to the electron fraction because the chemical heating from $\mathrm{H}^{+}$and $\mathrm{He}^{+}$are also essentially independent of the electron fraction. There are a number of fine points that could add or subtract 1-2 eV from this result, depending on the physical conditions and on the role of poorly understood processes. For example, this treatment of high-density chemical heating is an over-estimate in that no energy is assumed to be lost by vibrationally-excited molecules produced in the relevant chemical reactions, on the assumption that the density is high enough for collisional de-excitation to be effective. Similarly, we have ignored the uncertain possibility that $\sim 1.5 \mathrm{eV}$ of excitation energy of newly formed $\mathrm{H}_{2}$ molecules may be available for heating at high densities.

\section{Results}

In the previous sections we developed a theory for the heating of molecular gases exposed to cosmic rays and X-rays, with detailed consideration of chemical heating. To illustrate the magnitude of the various contributions to the heating, we list in Table 4 the maximum values for the case of zero electron fraction and densities high enough for vibrationallyexcited molecules to be collisionally de-excited; the electron energy is $E=1 \mathrm{keV}$. The chemical heating in Table 4 is based on somewhat arbitrary choices for the most abundant neutrals: $x(\mathrm{CO})=x(\mathrm{O})=x\left(\mathrm{H}_{2} \mathrm{O}\right)=10^{-4}$. Starting at the bottom, we see that the total 
maximum heating is $Q=18.7 \mathrm{eV}$ per ion pair; close to $50 \%$ of the energy expended per ion pair, which in this case is $W=36.8 \mathrm{eV}$. The largest fraction, about one half, is accounted for by chemical heating $(9.3 \mathrm{eV})$. This value is $\sim 20 \%$ larger than the value of $7.6 \mathrm{eV}$ obtained from reactions Eq. 17, $\mathrm{H}_{2}^{+}+\mathrm{H}_{2} \rightarrow \mathrm{H}_{3}^{+}+\mathrm{H}$, and Eq. 20, $\mathrm{H}_{3}^{+}+\mathrm{e} \rightarrow \mathrm{H}_{2}+\mathrm{H}$, along the lines of GL73. The next largest contribution, $5.2 \mathrm{eV}$, comes from vibrationally excited $\mathrm{H}_{2}$ molecules, produced by direct collisional excitation or following the excitation of the $B$ and $C$ states. Elastic scattering, rotational excitation, and dissociation account for the rest, or $\sim 20 \%$.

The values in Table 4 are based on several assumptions: the electron fraction is very small, the density very high and the abundances of $\mathrm{C}, \mathrm{H}_{2} \mathrm{O}$ and $\mathrm{O}$ all equal to $10^{-4}$. The chemical heating appears to be insensitive to the electron fraction as long as Eq. 19 is satisfied. On the other hand, Coulomb collisions with ambient electrons begin to play a significant role in heating wherever $x_{\mathrm{e}}$ approaches the $10^{-4}$ level. This effect is automatically included in Eq. 7. The entries for vibrational heating in Table 4 are the most sensitive to density because very high densities are required to collisionally quench vibrationally excited $\mathrm{H}_{2}$ molecules, as discussed in Section 2.4. This restriction means that vibrational heating is ineffective in molecular clouds outside of pre-stellar cores. The chemical heating also depends weakly on the abundances of the neutral species. For example, if instead of the abundances used for the estimates in Table $4, x(\mathrm{CO})=x(\mathrm{O})=x\left(\mathrm{H}_{2} \mathrm{O}\right)=10^{-4}$, we choose $x(\mathrm{CO})=10^{-4}, x(\mathrm{O})=2 \times 10^{-4}$, and $x\left(\mathrm{H}_{2} \mathrm{O}\right)=0$, the chemical heating from $\mathrm{H}_{2}^{+}$increases by about $\sim 0.2 \mathrm{eV}$. Thus the chemical heating associated with the $\mathrm{H}_{2}^{+}$ion is reasonably robust in molecular regions at $\sim 8-9 \mathrm{eV}$.

Under certain circumstances, such as very low energy cosmic-rays or soft X-rays, the considerations of this paper need to be extended to electron energies $E<1 \mathrm{keV}$. Without attempting to treat this subject in detail, we can obtain a preliminary view of the situation from Table 5, which gives heating functions $Q$ and the energy to make an ion pair as a 
Table 4. Heating per Ion Pair ${ }^{\mathrm{a}}$

\begin{tabular}{lc}
\hline \hline \multicolumn{1}{c}{ Process } & $Q(\mathrm{eV})$ \\
\hline$Q_{\mathrm{el} / \mathrm{rot}}$ & 2.1 \\
$Q_{\text {diss }}$ & 2.1 \\
$Q_{\text {dir } / \text { vib }}$ & 2.8 \\
$Q_{\mathrm{BC} / \text { vib }}$ & 2.4 \\
$Q_{\text {chem }}\left(\mathrm{H}_{2}^{+}, \mathrm{H}_{2}\right)$ & 7.7 \\
$Q_{\text {chem }}\left(\mathrm{H}^{+}, \mathrm{H}_{2}\right)$ & 0.3 \\
$Q_{\text {chem }}\left(\mathrm{H}_{2}^{+}, \mathrm{He}^{+}\right)$ & 1.3 \\
\hline Total heating $Q$ & 18.7 \\
\hline
\end{tabular}

${ }^{\mathrm{a}} E=1 \mathrm{keV}$ 
function of electron energy. Many of the heating functions increase with decreasing energy, especially collisional excitation and dissociation. However the percentage of the energy to make an ion pair that goes into heating remains close to $50 \%$ down to $E=50 \mathrm{eV}$. Indeed, the following quantities vary by less than $10 \%$ for energies greater than $200 \mathrm{eV}$ : total heating $Q$, energy to make an ion pair $W$ and the percentage heating.

We can make the implications of the theory in this paper more concrete by estimating the heating for representative examples of interstellar and circumstellar matter that have significant amounts of $\mathrm{H}_{2}$. The estimates in Table 6 are based on the assumption that the electron energy is greater than $0.5 \mathrm{keV}$, corresponding to cosmic-ray proton energies greater than $1 \mathrm{MeV}$. For the well-observed line of sight towards $\zeta$ Per $\left(A_{\mathrm{V}}=1\right)$ (an early focus of the $\mathrm{H}_{3}^{+}$observations, Indriolo et al. 2007), we use the modeling results for many observed species by Shaw et al. (2008), who fit the cosmic-ray ionization rate per $\mathrm{H}_{2}$ with $\zeta_{2} \simeq 8 \times 10^{-16} \mathrm{~s}^{-1}$. The densities in this diffuse cloud are too low for vibrational heating. The estimate for elastic/rotational heating may be too high because the density and temperature in this cloud may not be large enough for significant quenching of rotational excitation, according to the discussion in Sec. 3.1. The chemical heating in this case comes from dissociative recombination because, according to Eq. 23, it dominates over ion reactions. All of the heating estimates for $\zeta$ Per have been reduced by $70 \%$ to take into account that atomic $\mathrm{H}$ has not been fully converted into $\mathrm{H}_{2}$. The entries under $\mathrm{H}_{3}^{+}$destruction in Table 6 indicate whether the chemical heating is dominated by dissociative recombination of $\mathrm{H}_{3}^{+}$("DR") or ionic reactions ("I").

For the case of a molecular cloud clump, dissociative recombination plays a role in the destruction of $\mathrm{H}_{3}^{+}$, but ion-molecule reactions are probably more important. Again, clumps are not dense enough for vibrational heating, and they also may not be warm enough to quench rotational excitation. For the inner region of a pre-stellar core, the density may 
Table 5. Heating $Q$ (in eV) per Ion Pair vs. Electron Energy

\begin{tabular}{|c|c|c|c|c|c|c|}
\hline Electron Energy $E(\mathrm{eV})$ & 30 & 50 & 100 & 200 & 500 & 1000 \\
\hline$Q_{\mathrm{el} / \mathrm{rot}}$ & 7.7 & 4.4 & 3.0 & 2.5 & 2.1 & 2.1 \\
\hline$Q_{\text {diss }}$ & 12.0 & 5.8 & 3.9 & 2.7 & 2.3 & 2.1 \\
\hline$Q_{\operatorname{dir} / \mathrm{vib}}$ & 10.9 & 6.1 & 4.0 & 3.3 & 2.9 & 2.8 \\
\hline$Q_{\mathrm{BC} / \mathrm{vib}}$ & 4.3 & 3.3 & 2.9 & 2.6 & 2.4 & 2.4 \\
\hline$Q_{\text {chem }}\left(\mathrm{H}_{2}^{+}, \mathrm{H}_{2}\right)$ & 8.6 & 8.3 & 8.0 & 7.8 & 7.7 & 7.7 \\
\hline$Q_{\text {chem }}\left(\mathrm{H}^{+}, \mathrm{H}_{2}\right)$ & 0 & 0.1 & 0.3 & 0.3 & 0.3 & 0.3 \\
\hline$Q_{\text {chem }}\left(\mathrm{H}_{2}^{+}, \mathrm{He}^{+}\right)$ & 0.2 & 0.5 & 0.8 & 1.0 & 1.2 & 1.3 \\
\hline Total heating $Q$ & 43.7 & 28.5 & 22.9 & 20.2 & 18.9 & 18.7 \\
\hline Energy to make an ion pair $W$ & 76.8 & 52.9 & 43.1 & 39.6 & 37.4 & 36.8 \\
\hline Number of ion pairs $N=E / W$ & 0.39 & 0.95 & 2.3 & 5.1 & 13.4 & 27.2 \\
\hline Percentage heating & 57 & 54 & 53 & 51 & 51 & 51 \\
\hline
\end{tabular}


be high enough for vibrational heating, but their low temperatures suggest that the elastic/rotational heating may be somewhat smaller than given in Table 6. The freeze-out of volatiles, including some CO, means that the chemical heating will probably be close to the value for dissociative recombination.

The densities in the inner molecular layer of a protoplanetary disk are often in excess of $10^{9} \mathrm{~cm}^{-3}$, and both rotational and vibrational heating should be effective. Because these regions are close to a stellar X-ray source, the X-ray ionization rates are much larger than the cosmic-ray rates for interstellar matter. Thus the electron fractions are relatively high at the top of the molecular region, $\sim 10^{-7}-10^{-6}$, and Eq. 23 indicates that dissociative recombination still plays a role in the chemical heating of the very dense inner regions. However, for sufficiently large vertical columns, the X-ray ionization is sufficiently reduced that ionic reactions dominate chemical heating.

All of the cases considered in Table 6 contain a significant if not an overwhelming fraction of $\mathrm{H}_{2}$. These rough estimates for the total heating per ion pair increase with the total density, and range from $\sim 10 \mathrm{eV}$ per ion pair for a moderately thick diffuse cloud like $\zeta$ Per to $\sim 18 \mathrm{eV}$ per ion pair for the densest regions. Perhaps the most important conclusion from these four examples is that cosmic-ray and X-ray heating are sensitive to the physical conditions, as expressed in the equations presented in Sections 3 and 4 . The values of $Q$ listed in Table 6 for various environments, combined with prescriptions for $\zeta_{\mathrm{H}}$ as function of the column density of the ambient gas (Padovani et al. 2009) and for the magnetic field intensity (Padovani \& Galli 2011), allow the determination of the cosmic-ray heating rate in clouds, cores, and protostellar disks. 
Table 6. X-ray and Cosmic-ray Heating (in eV) in Molecular Regions

\begin{tabular}{|c|c|c|c|c|}
\hline & $\begin{array}{c}\zeta \text { Per } \\
\text { diffuse cloud }\end{array}$ & $\begin{array}{l}\text { molecular cloud } \\
\text { clump }\end{array}$ & $\begin{array}{l}\text { prestellar core } \\
\text { inner region }\end{array}$ & $\begin{array}{c}\text { protoplanetary disk } \\
\text { active region at } 1 \mathrm{AU}\end{array}$ \\
\hline$n_{\mathrm{H}}\left(\mathrm{cm}^{-3}\right)$ & 80 & $10^{4}$ & $10^{7}$ & $10^{10}$ \\
\hline$T(\mathrm{~K})$ & $\simeq 60$ & 10 & 6 & 1000 \\
\hline$x_{\mathrm{e}}$ & $2 \times 10^{-4}$ & $10^{-7}$ & $10^{-9}$ & $10^{-6}$ \\
\hline $\mathrm{H}_{3}^{+}$destruction & $\mathrm{DR}^{*}$ & $\mathrm{DR}+\mathrm{I}^{*}$ & $\mathrm{DR}+\mathrm{I}$ & $\mathrm{DR}+\mathrm{I}$ \\
\hline$Q_{\mathrm{el} / \mathrm{rot}}(\mathrm{eV})$ & 4 & 2 & 2 & 2 \\
\hline$Q_{\text {vib }}(\mathrm{eV})$ & 0 & 0 & 5 & 5 \\
\hline$Q_{\text {diss }}(\mathrm{eV})$ & 1 & 2 & 2 & 2 \\
\hline$Q_{\text {chem }}(\mathrm{eV})$ & 5 & 9 & 8 & 9 \\
\hline Total heating $Q(\mathrm{eV})$ & 10 & 13 & 17 & 18 \\
\hline
\end{tabular}

${ }^{*}$ DR stands for dissociative recombination and I for ionic reactions. 


\section{Conclusion}

Dalgarno et al. (1999; DYL) made an extensive study of the energy loss of fast electrons in $\mathrm{H}$, He and $\mathrm{H}_{2}$, He gas mixtures. The electrons are at the heart of the interaction of cosmic rays and X-rays with interstellar and circumstellar matter. Although DYL analyzed essentially all excitation and ionization processes, they only discussed the heating from elastic collisions and rotational excitation of $\mathrm{H}_{2}$. Starting from the results in DYL, we have extended the scope of cosmic-ray and X-ray heating to include all of the relevant interactions. One of the main conclusions of this study is that heating by fast-electrons depends on the physical properties of the gas, i.e., on the abundance of electrons, $\mathrm{H}_{2}$ molecules, and heavy atoms and molecules, and also on the total density of hydrogen nuclei. The electron fraction is important because, once it exceeds a certain level, heating by collisions with ambient electrons becomes important. The electron abundance also determines whether the destruction of the $\mathrm{H}_{3}^{+}$ ions proceeds by dissociative recombination or ionic reactions, which affects the quantitative amount of chemical heating. Of course the $\mathrm{H}_{2} / \mathrm{H}$ abundance ratio is important because the diversity of the energy levels of $\mathrm{H}_{2}$ offers many more channels for energy loss than atomic H. The dependence on physical conditions means that X-ray and cosmic-ray heating cannot

be specified by a single number. This should be clear from the fact that in neutral atomic regions the heating efficiency is only $12 \%$, whereas in neutral molecular regions it can reach $50 \%$ at very high densities.

A wide range of values for the heating per ion pair can be found in the literature on molecular clouds, from $7 \mathrm{eV}$ (Stahler \& Palla 2004), to 20 eV (Goldsmith \& Langer 1978, Goldsmith 2001), but not necessarily for the reasons given here, e.g., in the above discussion of Table 6. Many authors adopt Goldsmith's value while others estimate intermediate values of 10-15 eV based on GL73, e.g., Maloney et al. (1996), Yusef-Zadeh et al. (2007), and Krumholz et al. (2011). As shown in Table 4, $13 \mathrm{eV}$ is a good choice for not too dense 
molecular gas.

Chemical heating also applies to regions exposed to far UV radiation (Dalgarno and Oppenheimer 1974). It was then considered by Barsuhn \& Walmsley (1977) and Clavel et al. (1978), who studied the chemical and thermal equilibrium in dark clouds exposed to far UV radiation and cosmic rays. Clavel et al. explicitly included the contribution of every reaction in their chemical network to heating. Chemical heating has also been widely used in the study of planetary atmospheres (e.g., Roble et al. 1987).

The results of this paper are based on the fact that roughly half of the energy generated by cosmic rays and X-rays comes goes into ionization of the gas and roughy half into its excitation, more specifically according to the way the individual processes are treated by DYL. Roughly half of the gas heating comes from the reactions of various ions with the assumed mainly neutral gas. Potentially an equal amount of heating can arise from dissociation and from rotational and vibrational excitation, but the yield from excitation depends on whether the physical conditions are conducive to the quenching of the excited levels. If quenching is inefficient, the levels decay with the emission of radiation, which can escape or be absorbed by dust. This possibility is even more important for the excitation of the singlet levels of $\mathrm{H}_{2}$, e.g., the $B$ and $C$ levels, and the excitation of the $n=2$ level of $\mathrm{H}$ in atomic regions. We have not attempted to follow the fluorescent radiation to determine how much escapes and how much is absorbed. This is an important issue when considering the broader thermal properties of the gas. The treatment of the fluorescent radiation involves radiation transfer and depends on the properties of the dust, i.e., it is application specific. In contrast, our goal has been to treat one relatively well-defined part of the thermal problem of interstellar and circumstellar molecular gas.

In pursuing this goal, we have also ignored the direct interaction of X-rays and cosmicrays with the dust, of some interest because of the possibility that they may release fast 
electrons from the dust. Although this occurs, it is relatively unimportant. First of all, the dust cross section per $\mathrm{H}$ nucleus for the MRN distribution for the diffuse ISM is $1.6 \times$ $10^{-21} \mathrm{~cm}^{-2}$. For molecular clouds and disk atmospheres, it will be even smaller. The main

inelastic cross sections for a keV electron with $\mathrm{H}_{2}$ are typically several times $10^{-17} \mathrm{~cm}^{-2}$, so the probability that an incident X-ray or cosmic ray interacts with dust is roughly 1000 time less than with $\mathrm{H}_{2}$. And when this rare event does occur, no more than $1 \%$ goes into in photoelectrons, as shown by Dwek and Smith (1996) for the EUV/Xray bands.

\section{Acknowledgements}

We are indebted to Máté Ádámkovics for testing the heating terms described in this paper in a thermal-chemical code for protoplanetary disks. One of us (A.E.G.) acknowledges support from NASA grant NNG06GF88G (Origins) and NASA grant 1367693 (Herschel DIGIT). The authors thank an anonymous referee for a careful reading and thoughtful comments. 


\section{REFERENCES}

Abgrall, H., Roueff, E., Liu, X., Shemansky, D. E. 1997, ApJ, 481, 557

Balakrishnan, N., Forrey, R. C., Dalgarno, A. 1999, ApJ, 514, 520

Barsuhn, J., Walmsley, C. M. 1977, A\&A, 54, 345

Bates, D. R. \& Griffing, G. 1953, Proc. Phys. Soc., A66, 961

Clavel, J., Viala, Y. P., Bel, N. 1978, A\&A, 65, 435

Crapsi, A., Caselli, P., Walmsley, M. C., Tafalla, M. 2007, A\&A, 470, 221

Cravens, T. E., Victor, G. A., Dalgarno, A. 1975, P\&SS, 23, 1059

Cravens, T. E., Dalgarno, A. 1978, ApJ, 219, 750

Dalgarno, A., Oppenheimer, M. 1974, ApJ, 192, 597

Dalgarno, A., Yan, M., Liu, W. 1999, ApJS, 125, 237 (DYL)

Dwek, E., Smith, R. K. 1996, ApJ, 459, 686

Everett, J. E., Zweibel, E. G. 2011, ApJ 738, 60

Galli, D., Walmsley, M., Gonçalves, J. 2002, A\&A, 394, 275

Glassgold, A. E., Langer, W. D. 1973, ApJ 186, 859 (GL73)

Goldsmith, P. F.,Langer, W. D. 1978, ApJ , 222, 881

Goldsmith, P. F. 2001, ApJ 557, 736

Henry, R. J. W., McElroy, M. B. 1969, J. Atm. Sci., 26, 912

Hooper, J. W., McDaniel, E. W., Martin, D. W., Harmer, D. S. 1961. Phys. Rev., 131, 1123 
Indriolo, N., Geballe, T. R., Oka, T, McCall, B. J. 2007, ApJ, 671, 1736

Indriolo, N., Fields, B. D., McCall, B. J. 2009, ApJ, 694, 257

Indriolo, N., McCall, B. J. 2012, ApJ, 745, 911

Jasche, J., Ciardi, B., Enßlin, T. A. 2007, MNRAS, 380, 417

Krumholz, M. R., Leroy, A. K., McKee, C. F. 2011, ApJ, 731, 25

Le Bourlot, J, Pineau des Forêts \& Flower, D. R. 1999, MNRAS, 305, 802

Lee, T.-G., Balakrishnan, N., Forrey, R. C., Stancil, P. C., Shaw, G., Schultz, D. R., Ferland, G. J. 2008, ApJ, 689, 1105

Maloney, P. R., Hollenbach, D. J., Tielens, A. G. G. M. 1996, ApJ, 466, 561

Opal, C. B., Peterson, W. K. \& Beatty, E. C. 1971, J. Chem. Phys, 55, 4100

Pagani, L., Bacmann, A., Cabrit, S., Vastel, C. 2007, A\&A, 467, 179

Padovani, M., Galli, D., Glassgold, A. E. 2009, A\&A, 501, 619

Padovani, M., Galli, D. 2011, A\&A, 530, A109

Pineda, J. L., Bensch, F. 2007, A\&A, 470, 615

Rimmer, P. B., Herbst, E., Morata, O., Roueff, E. 2012, A\&A, 537, A7

Roble, R. G., Ridley, E. C., Dickinson, R. E. 1987, J. Geophys. Res., 92, 8745

Roser, J. E., Swords, S., Vidali, G., Manicò, G., Pirronello, V. 2003, ApJL, 596, L55

Schneider, I. F., Dulieu, O., Giusti-Suzor, A., Roueff, E. 1994, ApJ, 424, 983 (errata in ApJ, $486,580)$ 
Shaw, G., Ferland, G. J., Srianand, R., et al. 2008, ApJ, 675, 405

Shaw, G., Ferland, G. J., Henney, W. J., et al. 2009, ApJ, 701, 677

Stahler, S. W., Palla, F. 2004, Sec. 7.2.1, "The Formation of Stars". (Wiley-VCH; Weinheim)

Suchkov, A., Allen, R. J., Heckman, T. M. 1993, ApJ, 413, 542

Sundström, G. Mowat, J. R., Danared, H. et al. 1994, Science, 263, 785

Theard, L. P., Huntress, W. T. 1974, J. Chem. Phys., 60, 2840

Tielens, A. G. G. M. 2000, in "The Physics and Chemistry of the Interstellar Medium" (Cambridge)

Tiné, S., Lepp, S., Gredel, R., Dalgarno, A. 1997, ApJ, 481, 282

Wrathmall, S. A., Gusdorf, A, Fower, D.R. 2011, MNRAS, 382, 133

Yusef-Zadeh, F., Wardle, M., Roy, S. 2007, ApJL, 665, L123

\section{A. Chemical Heating in Atomic Regions}

Table A provides the same information on the energy partitions for an atomic $\mathrm{H}, \mathrm{He}$ mixture as Table 1 does for a $\mathrm{H}_{2}$, He mixture. Although less energy is expended in ion production (because of the smaller ionization potential of $\mathrm{H}$ compared to that for $\mathrm{H}_{2}$ ), the total number of ions is essentially unchanged at 27.2 ion pairs per keV. 
Table A. DYL Energy Partitions for a He, H mixture, $x_{\mathrm{e}}=0$ and $E=1 \mathrm{keV}$

\begin{tabular}{|c|c|c|c|c|}
\hline Process & $\begin{array}{c}W \\
(\mathrm{eV})\end{array}$ & $1 \mathrm{keV} / W$ & $\begin{array}{c}\text { Specific energy } \\
(\mathrm{eV})\end{array}$ & $\begin{array}{c}\text { Total energy } \\
(\mathrm{eV})\end{array}$ \\
\hline $\mathrm{H}^{+}$ & 39.8 & 25.1 & 13.60 & 342 \\
\hline $\mathrm{He}^{+}$ & 487 & 2.05 & 24.6 & 51 \\
\hline $\mathrm{He}^{2+}$ & 16400 & 0.061 & 54.4 & 3 \\
\hline Total Ion production & & & & 396 \\
\hline $\mathrm{H} 2{ }^{1} \mathrm{~S}$ & 267 & 3.75 & 10.2 & 38 \\
\hline $\mathrm{H} 2^{1} \mathrm{P}$ & 33.1 & 30.2 & 10.2 & 308 \\
\hline $\mathrm{H} n=3$ & 191 & 5.24 & 12.1 & 6 \\
\hline $\mathrm{H} n>3$ & 236 & 4.24 & 13.2 & 56 \\
\hline Total H excitation & & & & 408 \\
\hline $\mathrm{He} 2{ }^{1} \mathrm{~S}$ & 17300 & 0.058 & 20.6 & 1.2 \\
\hline $\mathrm{He} 2^{1} \mathrm{P}$ & 2080 & 0.481 & 21.2 & 10.2 \\
\hline He $2^{3} \mathrm{~S}$ & 50700 & 0.020 & 19.8 & 0.39 \\
\hline $\mathrm{He} 2^{3} \mathrm{P}+n>2^{3}$ & 30800 & 0.032 & 21.0 & 0.68 \\
\hline He $n>2^{1}$ & 3790 & 0.264 & 23 & 6.1 \\
\hline Total He excitation & & & & 19 \\
\hline Total excitation & & & & 427 \\
\hline Heating & & & & 117 \\
\hline Grand total & & & & 940 \\
\hline
\end{tabular}

The heating of an atomic region is simpler to treat than a molecular region because of the absence of rotational and vibrational excitation. The direct heating by elastic scattering is usually important. We ignore the radiation emitted following the electronic excitation of the levels of $\mathrm{H}$ and $\mathrm{He}$ in Table A, and focus here on the chemical heating. 
In addition to radiative recombination,

$$
\mathrm{e}+\mathrm{H}^{+} \rightarrow \mathrm{H}+h \nu, \quad \alpha\left(\mathrm{H}^{+}\right) \simeq 2.12 \times 10^{-10} T^{-0.73} \mathrm{~cm}^{3} \mathrm{~s}^{-1},
$$

$\mathrm{H}^{+}$can be destroyed by radiative attachment to $\mathrm{H}$ to form $\mathrm{H}_{2}^{+}$,

$$
\mathrm{H}^{+}+\mathrm{H} \rightarrow \mathrm{H}_{2}^{+}+h \nu, \quad k_{\mathrm{ra}} \simeq 2.10 \times 10^{-23} T^{1.80} \mathrm{~cm}^{3} \mathrm{~s}^{-1} .
$$

The rate coefficient for radiative association is especially small, and the probability to form $\mathrm{H}_{2}^{+}$this way is given by,

$$
P\left(\mathrm{H}^{+}, \mathrm{H}_{2}^{+} ; \mathrm{H}\right)=\frac{k_{\mathrm{ra}} x(\mathrm{H})}{k_{\mathrm{ra}} x(\mathrm{H})+\alpha x_{\mathrm{e}}}=\frac{x(\mathrm{H})}{x(\mathrm{H})+\left(\alpha / k_{\mathrm{ra}}\right) x_{\mathrm{e}}} .
$$

In order for radiative recombination to be important, the electron fraction must approach the value,

$$
\frac{k_{\mathrm{ra}}}{\alpha} \simeq 10^{-13} T^{2.53}
$$

This requirement is relatively easy to satisfy, e.g., $k_{\mathrm{ra}} / \alpha \sim 10^{-8}$ at $100 \mathrm{~K}$ and $\sim 10^{-4}$ at $4000 \mathrm{~K}$.

The energetics of the two processes are different. In both cases the radiation is lost or absorbed by dust grains and will be ignored. In an atomic $\mathrm{H}$ region the newly formed $\mathrm{H}_{2}^{+}$ can charge-exchange with $\mathrm{H}$ to form $\mathrm{H}_{2}$,

$$
\mathrm{H}_{2}^{+}+\mathrm{H} \rightarrow \mathrm{H}_{2}+\mathrm{H}^{+}, \quad k=6.4 \times 10^{-10} \mathrm{~cm}^{3} \mathrm{~s}^{-1}
$$

This reaction can provide up to $q\left(\mathrm{H}^{+} ; \mathrm{H}\right)=I P\left(\mathrm{H}_{2}\right)-I P\left(\mathrm{H}_{2}^{+}\right)=1.83 \mathrm{eV}$ in heating, where IP stands for ionization potential, but it has to compete with dissociative recombination, Eq. 17, which has a branching ratio,

$$
B_{\mathrm{e}}=\frac{\beta x_{\mathrm{e}}}{\beta x_{\mathrm{e}}+k x(\mathrm{H})}=\frac{(\beta / k) x_{\mathrm{e}}}{(\beta / k) x_{\mathrm{e}}+x(\mathrm{H})} .
$$

The ratio $k / \beta=5.33 \times 10^{-3} T^{0.4}$ determines the electron fraction where dissociative recombination is important, i.e., in regions where $x_{\mathrm{e}} \sim 0.01$. 
We follow Section 3 and express the heating per ion pair for atomic regions due to the $\mathrm{H}^{+}$ion as,

$$
Q_{\text {chem }}\left(\mathrm{H}^{+} ; \mathrm{H}\right)=\frac{x(\mathrm{H})}{x(\mathrm{H})+x\left(\mathrm{H}_{2}\right)} F\left(\mathrm{H}^{+} ; \mathrm{H}\right) P\left(\mathrm{H}^{+}, \mathrm{H}_{2}^{+} ; \mathrm{H}\right)\left[B_{\mathrm{H}} q_{\mathrm{H}}\left(\mathrm{H}^{+} ; \mathrm{H}\right)+B_{\mathrm{e}} q_{\mathrm{e}}\left(\mathrm{H}^{+} ; \mathrm{H}\right)\right],
$$

where $F\left(\mathrm{H}^{+} ; \mathrm{H}\right)$ is the fraction of all ions that are $\mathrm{H}^{+} ; P\left(\mathrm{H}^{+}, \mathrm{H}_{2}^{+} ; \mathrm{H}\right)$ is the probability that an $\mathrm{H}^{+}$ion is converted into $\mathrm{H}_{2}^{+} ; q_{\mathrm{H}}\left(\mathrm{H}^{+} ; \mathrm{H}\right)=1.83 \mathrm{eV}$ is the net heating from reactions Eqs. A2 and A5, $B_{\mathrm{H}}$ is the branching ratio for reaction Eq. A5,

$$
B_{\mathrm{H}}=\frac{k x(\mathrm{H})}{k x(\mathrm{H})+\beta x_{\mathrm{e}}}=\frac{k x(\mathrm{H})}{x(\mathrm{H})+(\beta / k) x_{\mathrm{e}}}
$$

and $q_{\mathrm{e}}\left(\mathrm{H}^{+} ; \mathrm{H}\right)=11.0 \mathrm{eV}$ is the net heating from radiative charge exchange, Eq. A2, followed by dissociative recombination, Eq. 17. From Table A, we find that the fraction of $\mathrm{H}^{+}$ions $F\left(\mathrm{H}^{+} ; \mathrm{H}\right)=0.92$ for the parameters in that table $\left(E=1 \mathrm{keV}\right.$ and $\left.x_{\mathrm{e}}=0\right)$; it increases slowly with decreasing electron energy.

The maximum chemical heating per ion pair coming from $\mathrm{H}^{+}$depends on how the $\mathrm{H}_{2}^{+}$it generates is destroyed: $1.7 \mathrm{eV}$ by charge exchange and $10.1 \mathrm{eV}$ by dissociative recombination, with the latter only occurring for relatively large electron fractions. However in most H I regions, the dominant factor is the probability of forming $\mathrm{H}^{+}, P\left(\mathrm{H}^{+}, \mathrm{H}_{2}^{+} ; \mathrm{H}\right)$ in Eq. A7, which can greatly reduce $Q_{\text {chem }}\left(\mathrm{H}^{+} ; \mathrm{H}\right)$, simply because radiative association in this case is such a weak process. Thus chemical heating in atomic $\mathrm{H}$ regions is usually negligible.

In addition to radiative recombination,

$$
\mathrm{e}+\mathrm{He}^{+} \rightarrow \mathrm{He}+h \nu, \quad \alpha\left(\mathrm{He}^{+}\right) \simeq 2.12 \times 10^{-10} T^{-0.73} \mathrm{~cm}^{3} \mathrm{~s}^{-1},
$$

$\mathrm{He}^{+}$can be destroyed by radiative charge exchange,

$$
\mathrm{He}^{+}+\mathrm{H} \rightarrow \mathrm{H}^{+}+\mathrm{He}+h \nu, \quad k_{\text {chex }} \simeq 1.6 \times 10^{-16} T^{0.50} \mathrm{~cm}^{3} \mathrm{~s}^{-1},
$$

and by radiative association to form $\mathrm{HeH}^{+}$,

$$
\mathrm{He}^{+}+\mathrm{H} \rightarrow \mathrm{HeH}^{+}+h \nu, \quad k_{\mathrm{ra}} \simeq 1.33 \times 10^{-14} T^{-0.37} \mathrm{~cm}^{3} \mathrm{~s}^{-1} .
$$


All of these radiative processes are weak, although the last two are not as weak as the corresponding reactions just discussed that start from $\mathrm{H}^{+}+\mathrm{He}$. These reactions have the potential to provide greater heating because of the larger energy of the $\mathrm{He}^{+}$ion, except that much of the available energy is lost in radiation. We therefore ignore radiative recombination and focus on radiative charge exchange, Eq. A10, and radiative association, Eq. A11. The former process generates $I P(\mathrm{He})-I P(\mathrm{H})=11.0 \mathrm{eV}$ in recombination radiation rather than gas heating. The heating from the formation of the $\mathrm{HeH}^{+}$ion, discussed in the previous section, has a maximum value $1.83 \mathrm{eV}$. But this value must be reduced by the fraction of $\mathrm{He}^{+}$which, according to Table A for $E=1 \mathrm{keV}$, is $F\left(\mathrm{He}^{+}, \mathrm{H}\right)=0.075$. It may also be reduced by the branching ratio for radiative association,

$$
B_{\mathrm{ra}}\left(\mathrm{He}^{+}\right)=\frac{k_{\mathrm{ra}}}{k_{\mathrm{ra}}+k_{\text {chex }}+\alpha x_{\mathrm{e}}},
$$

which is often less than unity. For example, in warm regions charge exchange is more important than radiative association, and radiative recombination is competitive with the other reactions for $x_{\mathrm{e}}>10^{-3}$. Thus we cannot expect the chemical heating from $\mathrm{He}^{+}$to be any more than $0.14 \mathrm{eV}$, and thus negligible, as we found also for the $\mathrm{H}^{+}$ion. 